\title{
Muscle wasting associated with pathologic change is a risk factor for the exacerbation of joint swelling in collagen-induced arthritis in cynomolgus monkeys
}

Naoto Horai ${ }^{1,2 *}$, Takaharu Nagaoka ${ }^{2}$, Itsuro Higuchi ${ }^{3}$, Hayato Kasai ${ }^{2}$, Takako Yoshioka ${ }^{4}$, Yoshihisa Umekita ${ }^{5}$, Koichiro Fukuzaki ${ }^{2}$, Ryoichi Nagata ${ }^{2}$, Atsuro Miyata ${ }^{1}$ and Kazuhiro Abeyama ${ }^{6}$

\begin{abstract}
Background: Not only joint destruction but also muscle wasting due to rheumatoid cachexia has been problem in terms of quality of life of patients with rheumatoid arthritis (RA). In the present study, we performed histopathological examination and assessed relationships between characteristic parameters relating to muscle and joint swelling in a collagen-induced arthritis (CIA) model using cynomolgus monkeys (CMs).

Methods: Female CMs were used and CIA was induced by twice immunizations using bovine type II collagen with Freund's complete adjuvant. Arthritis level was evaluated from the degree of swelling at the peripheral joints of the fore and hind limbs. Food consumption, body weight, and serum biochemical parameters were measured sequentially. Five or 6 animals per time point were sacrificed at 2, 3, 5 and 9 weeks after the first immunization to obtain quadriceps femoris specimens for histopathology. Pimonidazole hydrochloride was intravenously administered to determine tissue hypoxia in skeletal muscle.

Results: Gradual joint swelling was observed and the maximum arthritis score was noted at Week 5. In histopathology, necrosis of muscle fiber in the quadriceps femoris was observed only at Week 2 and the most significant findings such as degeneration, atrophy, and regeneration of muscle fiber were mainly observed at Week 5. Food consumption was decreased up to Week 4 but recovered thereafter. Body weight decreased up to Week 5 and did not completely recover thereafter. A biphasic increase in serum cortisol was also observed at Weeks 2 and 5. Histopathology showed that muscle lesions were mainly composed of degeneration and atrophy of the muscle fibers, and ATPase staining revealed that the changes were more pronounced in type II muscle fiber than type I muscle fiber. In the pimonidazole experiment, mosaic pattern in skeletal muscle was demonstrated in the intact animal, but not the CIA animal. Increased arthritis score was accompanied by a decrease in serum creatinine, a marker that reflects muscle mass.
\end{abstract}

Conclusions: Muscle wasting might exacerbate joint swelling in a collagen-induced arthritis model of cynomolgus monkeys.

Keywords: Collagen-induced arthritis, Rheumatoid arthritis, Cynomolgus monkey, Muscle wasting, Hypoxia, Steroid, Histopathology, Muscle

\footnotetext{
* Correspondence: horai-naoto@snbl.co.jp

'Department of Pharmacology, Kagoshima University Graduate School of

Medical and Dental Sciences, 8-35-1 Sakuragaoka, Kagoshima 890-8544,

Japan

${ }^{2}$ Shin Nippon Biomedical Laboratories, Ltd. Drug Safety Research

Laboratories (SNBL DSR), 2438 Miyanoura, Kagoshima 891-1394, Japan

Full list of author information is available at the end of the article
} 


\section{Background}

We have already established a collagen-induced arthritis (CIA) model using female cynomolgus monkeys (CMs) and have assessed therapeutic and preventive effects of new drugs for rheumatoid arthritis (RA) in this model [1-3]. A treatment with biological agents targeting tumor necrosis factor- $\alpha$ (TNF- $\alpha$ ) showed therapeutic effects in RA patients who were resistant to disease-modifying antirheumatic drugs (DMARDs) [4,5]. Employing primate models is considered to have many advantages for the prediction of drug effects with clinical application because of the similarities in drug metabolism between humans and CMs [6-8].

Rats and mice arthritis models induced by treatment with collagen (CIA) or adjuvant (AIA) are well known as animal models for chronic inflammation and RA [9-13]. However, it is difficult to assess toxicological and pharmacological aspects of biologics for humans with these rodent models, because rodent arthritis is not chronic, and swelling including soft tissue at peripheral part from wrist and ankle rather than joint structure itself is often assessed $[14,15]$. Additionally, humanized antibody drugs are easily rendered ineffective by rapidly produced neutralizing antibodies in rodents [16]. On the other hand, joint swelling in the CM CIA model is chronic, and the production of neutralizing antibodies for antibody drugs is negligible in $\mathrm{CM}$ in comparison with that in rodents $[17,18]$.

Some epidemiological studies revealed a higher risk of cardiovascular disease $[19,20]$ and muscle wasting $[21,22]$ in RA patients compared with the general population. As well as the characteristic joint disorders, muscle dysfunction is a common and clinically intractable complication which also negatively affects prognosis and/or quality of life in RA $[23,24]$. The CIA model in CMs is also characterized by a body weight loss and cachexia associated with a loss of skeletal muscle.

RA is characterized by an inflammatory joint disease with chronic hypoxia and inflammatory cytokine production. Previous studies revealed hypoxia in the inflammatory tissues around the synovial membrane during RA development $[25,26]$. Although the linkage between hypoxia and inflammation is not clear, the possibility that tissue hypoxia and its resultant extracellular high mobility group box 1 (HMGB1) play an important role in arthritis development has been indicated [27].

Glucocorticoids regulate muscle metabolism and a number of steroids are used for patients with diverse diseases including RA $[28,29]$. However, it is known that cortisol, a representative intrinsic glucocorticoid in humans and monkeys, is induced by inflammation, and that muscle wasting due to the increases in the steroids is a critical clinical issue [30].

We hypothesized that body weight/muscle loss accompanied by inflammatory changes may have a role in the development of joint swelling as a representative pathosis in CIA. In the present study, we examined joints and skeletal muscle histopathologically to characterize the "arthritis-associated myopathy" and investigated the relation between muscle wasting and joint swelling, a major symptom of CIA.

\section{Methods}

\section{Animals}

Twenty-seven female cynomolgus monkeys (CMs) (Macaca fascicularis) with ages of 3 to 5 years were obtained from Guangdong Scientific Instruments \& Materials Import/Export Corporation (Guangzhou, China), Wing freight agent Co., Ltd. (Beijing, China), China National Scientific Instruments \& Materials Import/Export Corporation (Beijing, China), and Gaoyao Kangda Laboratory Animals Science \& Technology Co., Ltd. (Guangdong, China). Twenty-two CMs were used for sequential histopathology and a part of the quadriceps femoris was collected for special staining (NADH-TR and ATPase staining) from 3 of the animals that were necropsied at Week 5 after the first immunization. Additionally, arthritis was induced in 1 animal, which was then allocated for a pimonidazole dosing study. One intact animal was used for the pimonidazole dosing study as a control animal. The $22 \mathrm{CMs}$ to be used for histopathology were weighed using an electronic balance (HP-40 K, A \& D Co., Ltd.) once a week throughout the experiment. Approximately $108 \mathrm{~g}$ of solid food (Teklad Global Certified 25\% Protein Primate Diet, Harlan Sprague Dawley Inc.) was provided to each animal daily. Food consumption was calculated daily from the amounts of food supplied and remaining, and averaged daily food consumption was calculated for each week. Water was available ad libitum from an automatic supply (Edstrom Industries, Inc.).

\section{Animal welfare}

All procedures for animals were approved by the Intuitional Animal Care and Use Committee of SNBL and were performed in accordance with standards published by the National Research Council (Guide for the Care and Use of Laboratory Animals, NIH OACU) of the National Institutes of Health Policy on Human Care and Use of Laboratory Animals. Additionally, the animals used in this model received special treatments to moderate emaciation. In accordance with these standards, every effort was made to ensure that the subjects were free of pain and discomfort.

\section{Arthritis induction}

Bovine type II collagen (CII) $(4 \mathrm{mg} / \mathrm{mL}$, Collagen Research Center, Tokyo, Japan) was used. The CII solution and Freund's complete adjuvant (FCA) (Becton Dickinson, Grayson, GA, USA) were mixed in equal proportions using a syringe. Each $\mathrm{CM}$ was anaesthetized by intramuscular 
injection of $10 \mathrm{mg} / \mathrm{kg}$ ketamine and intracutaneously injected $2 \mathrm{~mL}$ of the emulsion on the back. The second immunization with CII and FCA was conducted 3 weeks after the first immunization, in the same manner $[1,2]$.

\section{Observation of swelling at the joints}

Arthritis level was evaluated by monitoring the degree of swelling at the metacarpophalangeal, proximal interphalangeal, and distal interphalangeal joints, and the wrist, ankle, elbow, and knee (total 64 joints) at Weeks 2, 3, 4, 5, 7 and 9 after the first immunization. Each joint was assessed in accordance with the evaluation criteria shown as follows: Score 0, No abnormality; Score 1, Swelling not visible but can be determined by touch; Score 2, Swelling slightly visible and can be confirmed by touch; Score 3, Swelling clearly visible; Score 4, Rigidity of the joints. The arthritis score for each animal was designated as the total score of individual joints.

\section{Blood chemistry}

Blood was drawn from the femoral vein at Weeks 2, 3, 4, 5, 7 and 9 after the first immunization, and serum was obtained by centrifugation (room temperature, $1710 \mathrm{~g}$ for 15 minutes). Creatine phosphokinase (CPK), creatinine and C-reactive protein (CRP) were determined by an automatic analyzer (JCA-BM8, JEOL Co., Ltd., Tokyo, Japan).

\section{Measurement of serum cytokines and cortisol}

Serum interleukin-6 (IL-6), IL-2, IL-4, IL-5, TNF, and IFN- $\gamma$ at Weeks 2, 3, 4, 5, 7 and 9 after the first immunization were determined with nonhuman primate Th1/Th2 Cytokine CBA kit (BD Biosciences, San Diego, CA, USA). Cortisol concentrations in serum obtained in the morning on each day were measured by radioimmunoassay with DPC-Cortisol kit (Siemens Healthcare Diagnostics, Los Angeles, CA, USA).

\section{Histopathology}

At Weeks 2, 3, 5 or 9 after the first immunization, 5 or 6 animals/time point were euthanized by exsanguination under anesthesia by an intravenous injection of a solution of sodium pentobarbital (Tokyo Chemical Industry Co., Ltd., $64.8 \mathrm{mg} / \mathrm{mL}, 0.4 \mathrm{~mL} / \mathrm{kg}$ ) and necropsied. The quadriceps femoris muscle from the center of lateral vastus on the left side was collected from each animal, and a half of the collected tissue was fixed in $10 \mathrm{v} / \mathrm{v} \%$ neutral buffered formalin and the remainder was quickly frozen in liquid nitrogen. The formalin-fixed tissue samples were embedded in paraffin, sectioned and stained with hematoxylin-eosin (HE). For electron microscopy, small pieces of the formalin-fixed muscle from 1 CIA animal with marked histopathological lesions were re-fixed with $3 \%$ glutaraldehyde and followed by a double fixation with $1 \%$ osmium tetroxide. Ultra-thin sections were prepared and double-stained with uranyl acetate and lead citrate, and examined with a transmission electron microscope (JEM-1200EX, JEOL Co., Ltd.). NADH-TR and ATPase staining was performed with cryosections of muscle collected at Week 5.

\section{Detection of hypoxia in the skeletal muscle with pimonidazole}

Hypoxic changes in the left quadriceps femoris were visualized using a hydroxyprobe-1 kit (Chemicon International, Temecula, CA, USA) as follows. Pimonidazole hydrochloride, a novel hypoxic marker agent, was dissolved in sterile physiological saline and 2 monkeys (one was a CIA model animal and the other an intact control animal) were administered intravenously with the pimonidazole solution at a dose of $0.5 \mathrm{~g} / \mathrm{m}^{2}$ at Week 5 . At 18 hours after dosing, the animals were euthanized by exsanguination under anesthesia by an intravenous injection of the solution of sodium pentobarbital $(0.4 \mathrm{~mL} / \mathrm{kg})$. A piece of the quadriceps femoris muscle was collected from each animal, fixed in $10 \mathrm{v} / \mathrm{v} \%$ neutral buffered formalin, and embedded in paraffin. A hydroxyprobe-1 monoclonal antibody was used to stain pimonidazole adducts in the thin-sectioned tissues in accordance with the recommendations of the manufacturer.

\section{Statistical analysis}

Values are presented as the mean \pm SE. Differences between post- and pre-dosing values as presented in Figure 1 were statistically examined using a repeated measures ANOVA model. Statistical differences were evaluated at $5 \%$. The number of animals at each time point were as follows; $\mathrm{N}=22$ at pre-immunization and Weeks 1 and 2, $\mathrm{N}=17$ at Week $3, \mathrm{~N}=12$ at Weeks 4 and 5 , and $\mathrm{N}=6$ at Weeks 6 to 9. Pearson's correlation coefficients were calculated for parameters (arthritis score, serum creatinine, serum CPK, and body weight) as shown in Figure 2.

\section{Results}

\section{Arthritis induction and its characters}

Joint swelling was observed mainly at the fingers of right and left hands and feet after CII immunization. There were individual differences in the time of onset and severity of the swelling. The mean arthritis score increased from Week 3 and was elevated sequentially with a peak value of 68.9 at Week 7, and the score was maintained around the peak value until Week 9 (Figure 1A). X-ray findings such as joint space narrowing, bone atrophy, and architectural joint destruction were aggravated time-dependently until Week 9 (data not shown).

\section{Body weight and food consumption}

Accompanying the occurrence of arthritis, body weights decreased markedly at Week 4 and remained lower than 

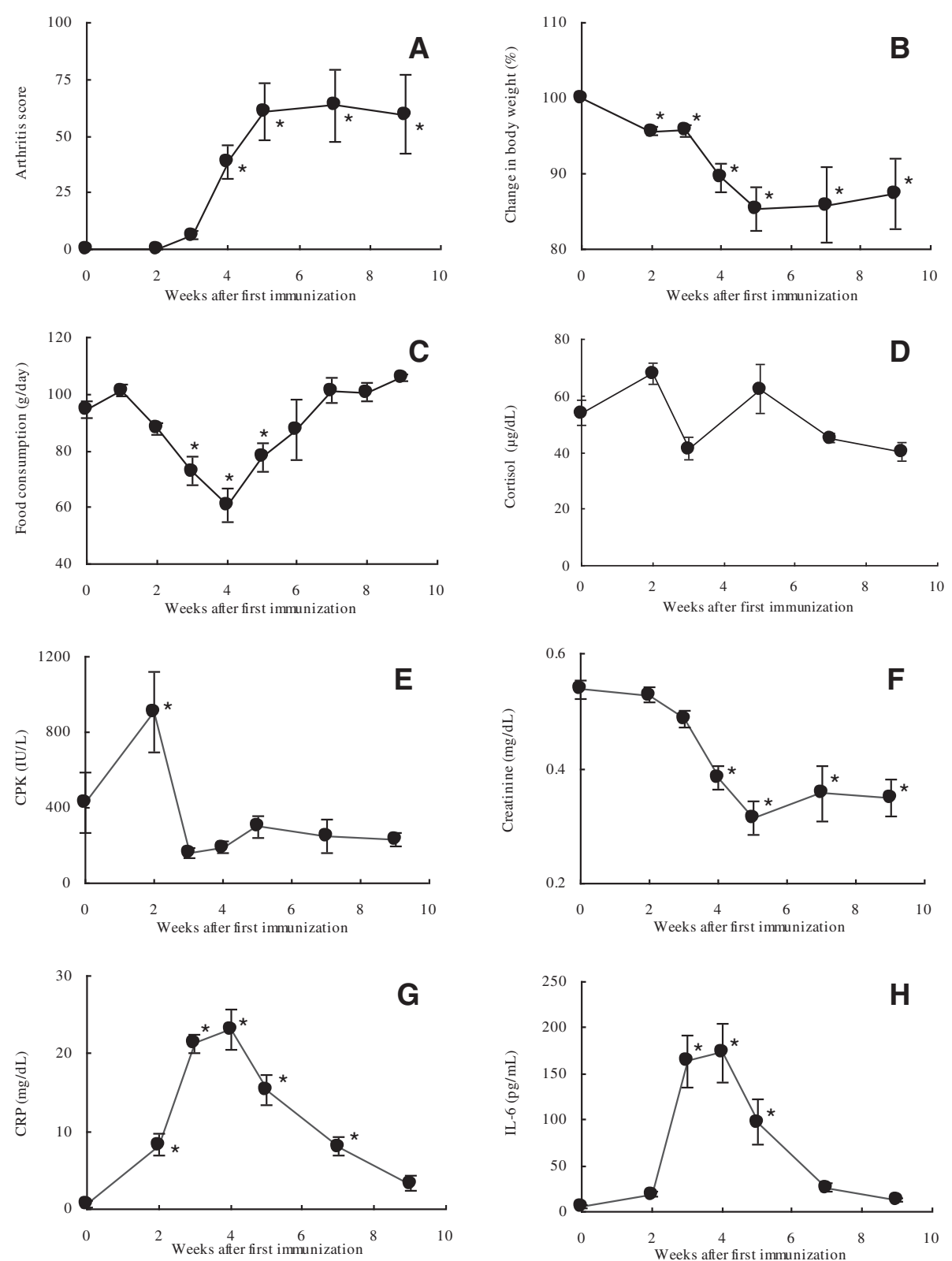

Figure 1 Sequential changes of the parameters in the process of arthritis induction. Changes in arthritis score (A), body weight (B), food consumption (C), serum cortisol concentration (D), creatine phosphokinase (E), creatinine (F), C-reactive protein (G), and interleukin-6 (H). Values are expressed as the mean \pm SEM. Differences between post- and pre-dosing values were statistically examined using a repeated measures ANOVA model. Statistical differences were evaluated at 5\%. $\mathrm{N}=22$ at pre-immunization and Weeks 1 and 2, N=17 at Week 3, N=12 at Weeks 4 and 5, and $\mathrm{N}=6$ at Weeks 6 to 9. Change in body weight (\%) was calculated as the percentage of weight relative to the weight at pre-immunization.

the value at pre-immunization until Week 9 (Figure 1B). Food consumption decreased time-dependently from the early phase of the induction period, and the most significant decrease in body weight was noted at Week 4, but food consumption recovered from the decrease to within the range of pre-immunization period by Week 7 (Figure 1C). The timing of recovery of food consumption was slightly sooner than that of body weight, and the time of transition was not consistent between these two parameters in the later phase of the induction period. These results support the previous observations that demonstrated that cachexia is not the result of a decrease in caloric intake [21].

\section{Blood chemistry and serum cytokine levels}

CPK was increased markedly at Week 2; however, the increase was transient and was followed by a decrease to 

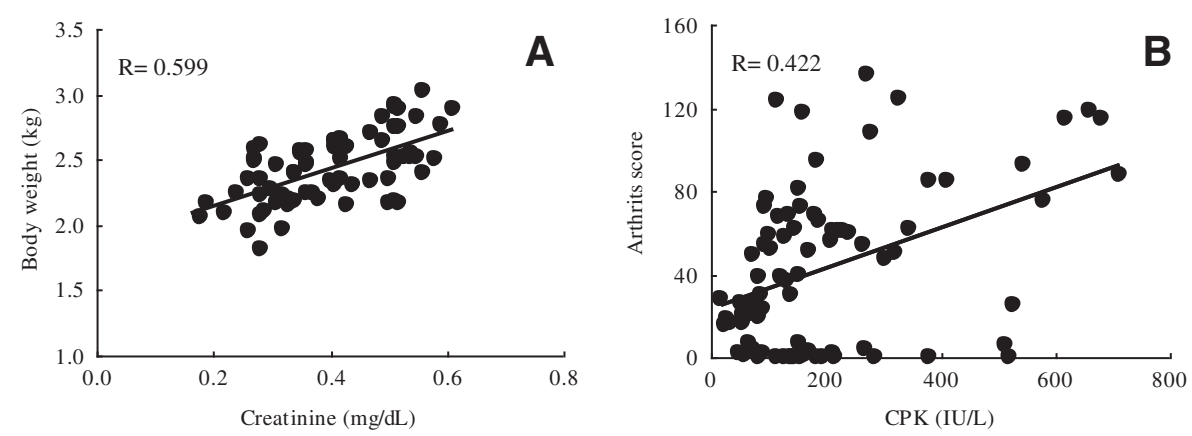

B
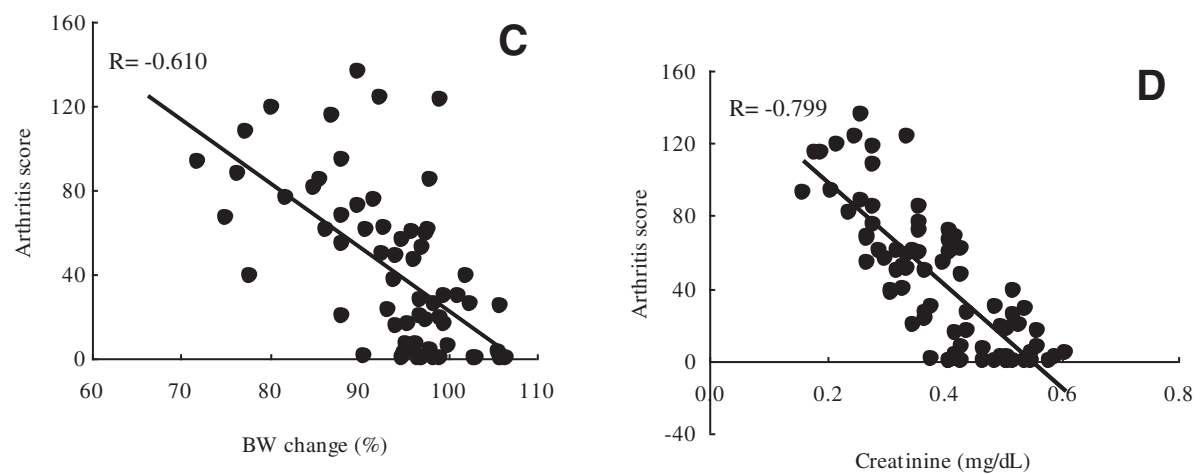

Figure $\mathbf{2}$ Correlation analysis. Relationships between serum creatinine level and body weight (A), creatine phosphokinase and arthritis score (B), change in body weight and arthritis score (C), and serum creatinine level and arthritis score (D). Pearson's correlation coefficients between the parameters (arthritis score, serum creatinine, serum CPK, and body weight) were calculated. The concentration of serum creatinine, a marker to reflect muscle mass, was positively correlated with body weight (A). CPK levels were not well related with arthritis score (B), but arthritis score showed a weak correlation with body weight change (C). Arthritis score showed a strong correlation with creatinine levels (D).

the pre-immunization level (Figure 1E). Creatinine decreased gradually from the early phase of induction period and reached nadir at Week 5, and no marked increase was noted until Week 9 (Figure 1F). Creatinine is known to be a marker which reflects muscle mass and to correlate with body weight. Both CRP and IL-6 increased up to Week 4, and then decreased time-dependently (Figures 1G, H). Serum TNF- $\alpha$ level was below the lower limit of quantification $(20 \mathrm{pg} / \mathrm{mL})$ in all animals at all time points, and no changes that were considered to be related to onset of arthritis were noted in the other cytokines (data not shown).

\section{Histopathological features}

The incidences of histopathological changes in the muscle fiber of the quadriceps femoris at each time point are summarized in Table 1. Degeneration, necrosis, or regeneration of muscle fiber was diagnosed, when a few mass or affected muscle bundles was observed in several scattered areas. Atrophy was diagnosed when width of the muscle bundle was smaller than half width of normal bundle. Necrosis and degeneration of the muscle fiber were seen in 2 and 1 of 5 animals at Week 2, respectively, but the incidences of degeneration and atrophy increased time-dependently until Week 5. Regeneration was seen at Week 5 and thereafter (Figures $3 \mathrm{~A}$ to E). In NADH-TR staining, there was a difference in stain ability between the muscle fiber types (intensely stained: type I muscle fiber, slightly stained: type II muscle fiber) in a naive animal (Figure 4A-1); however, the difference in stain ability was lost and irregularity in the size of muscle fibers was observed in the CIA animal (Figure 4A-2). ATPase staining showed that atrophy of type II muscle fiber was predominant when compared to that of the lesion in type I muscle fiber (Figure 4B-1: naive

Table 1 Frequency and percentage of animals with histopathological findings in muscle fiber

\begin{tabular}{|c|c|c|c|c|c|c|c|c|}
\hline & \multirow{2}{*}{\multicolumn{2}{|c|}{$\begin{array}{c}\text { Week } 2 \\
(N=5)\end{array}$}} & \multirow{2}{*}{\multicolumn{2}{|c|}{$\begin{array}{c}\text { Week } 3 \\
(N=5)\end{array}$}} & \multirow{2}{*}{\multicolumn{2}{|c|}{$\begin{array}{c}\text { Week } 5 \\
(N=6)\end{array}$}} & \multirow{2}{*}{\multicolumn{2}{|c|}{$\begin{array}{c}\text { Week } 9 \\
(N=6)\end{array}$}} \\
\hline & & & & & & & & \\
\hline & $\#$ & $\%$ & $\#$ & $\%$ & $\#$ & $\%$ & $\#$ & $\%$ \\
\hline Necrosis & 2 & 40 & 1 & 20 & 0 & 0 & 0 & 0 \\
\hline Degeneration & 1 & 20 & 3 & 60 & 6 & 100 & 5 & 83 \\
\hline Atrophy & 0 & 0 & 3 & 60 & 4 & 67 & 4 & 67 \\
\hline Regeneration & 0 & 0 & 0 & 0 & 3 & 50 & 1 & 17 \\
\hline
\end{tabular}

\#: Number of the animals with the histopathological finding. $\%$ : Percentage of the animals with the histopathological finding As for degeneration, necrosis, or regeneration of muscle fiber, when a few mass or affected muscle bundles were observed in several scattered areas, the finding was judged positive. Atrophy was diagnosed when width of the muscle bundle was smaller than half width of normal bundle. The number of animals with the above lesions was summarized in Table 1. 

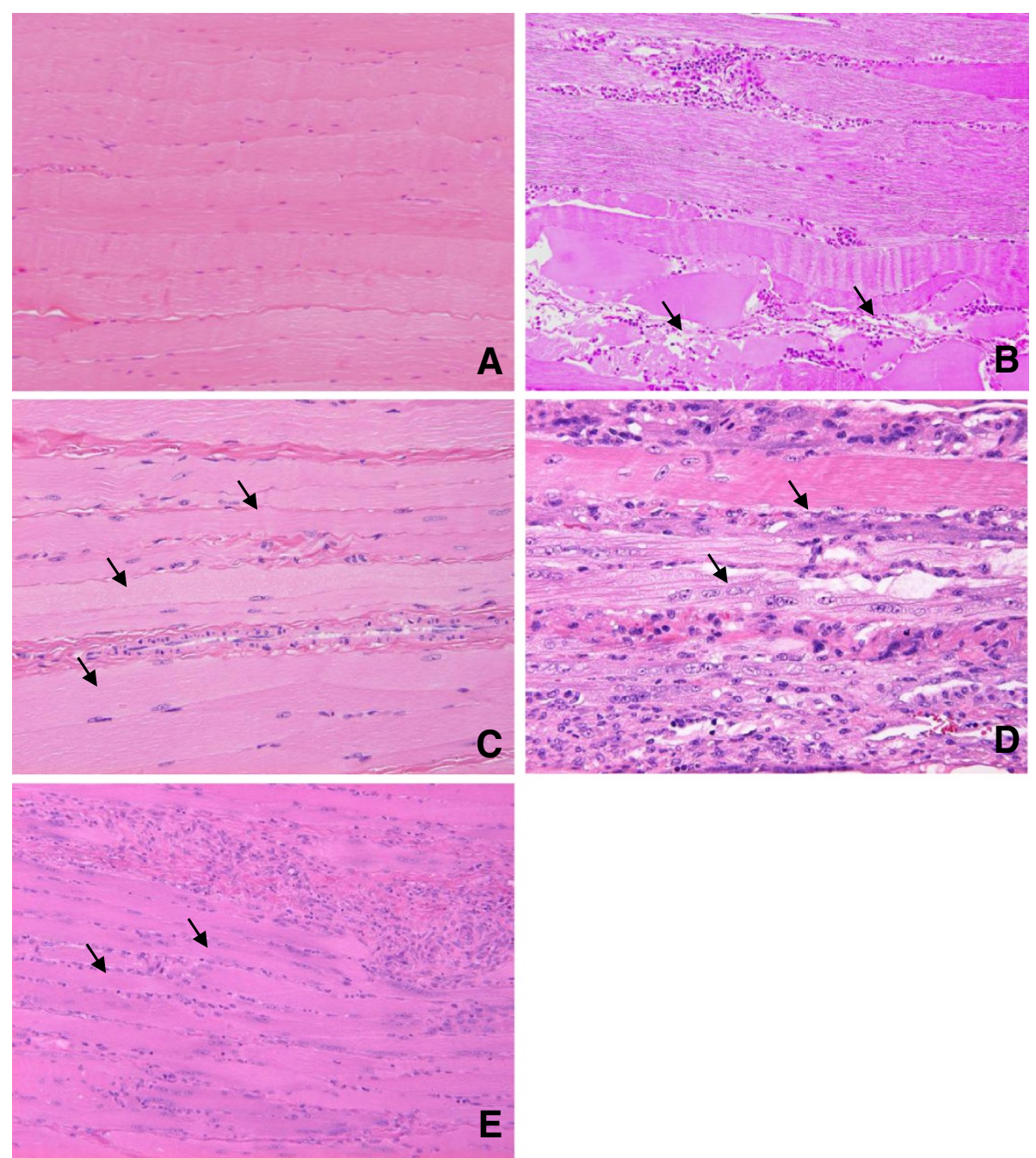

Figure 3 Sequential changes of histopathologic feature of quadriceps femoris in HE staining. A: naive animal, B: necrosis and degeneration ( $\uparrow$ ) of muscle fiber and inflammatory cell infiltration at Week 2, C: degeneration and atrophy ( $\uparrow$ ) of muscle fiber at Week 3, which is clearer than that at Week2, D: regeneration ( $\uparrow$ ) of muscle fiber at Week 5, E: regeneration and atrophy $(\uparrow)$ at Week 9 similar to, but a lesser degree to that at Week 5 (E).

animal, and Figure 4B-2: CIA animal). In electron microscopic observation, the number of mitochondria and vesicle increased with irregular alignment as the most characteristic change (Figures 5B, C) compared with a naive animal (Figure 5A).

\section{Detection of hypoxic change in femoral muscle}

The pimonidazole staining of the quadriceps femoris from 1 immunized and 1 non-immunized CM demonstrated a mosaic pattern of skeletal muscle in the naive animal (Figure 6A), and this was considered to reflect the normal distribution of type I (aerobic: unstained) and II (anaerobic: stained) muscle fibers. On the other hand, no mosaic pattern was observed in the quadriceps femoris of the CIA animal (Figure 6B), indicating that the normal distribution of the fibers had been changed in the muscle.

\section{Serum cortisol level}

Serum cortisol levels were elevated at Week 2 and dropped below the baseline at Week 3, and then were elevated again at Week 5 . The biphasic increase was considered attributable to twice immunizations (Weeks 0 and 3) with type II collagen. Thereafter, levels gradually decreased until Week 9 (Figure 1D).

\section{Relationships between arthritis score and muscle-related blood parameters}

Relationships between severity of swelling at the joints and blood parameters related to muscle (i.e. creatinine and CPK) were investigated. Serum creatinine is considered to depend on muscle mass, and its concentrations were positively correlated with body weight $(\mathrm{R}=0.599$, Figure $2 \mathrm{~A})$. Although CPK levels were not well related with arthritis 


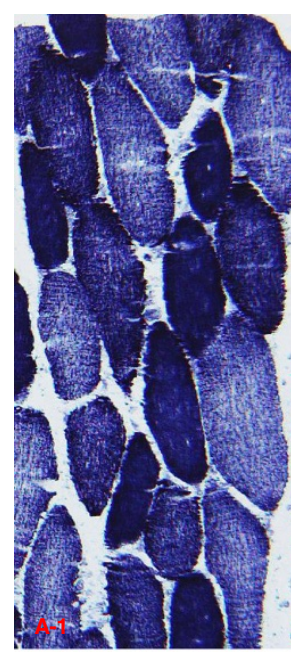

Naive

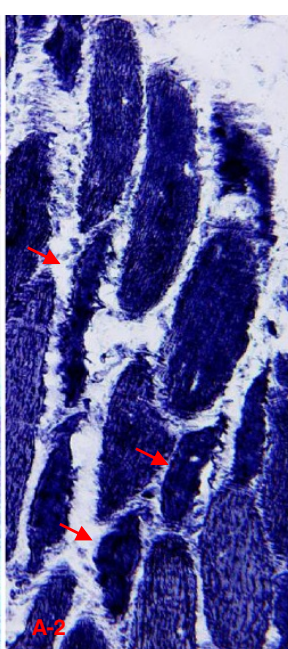

CIA

NADH-TR

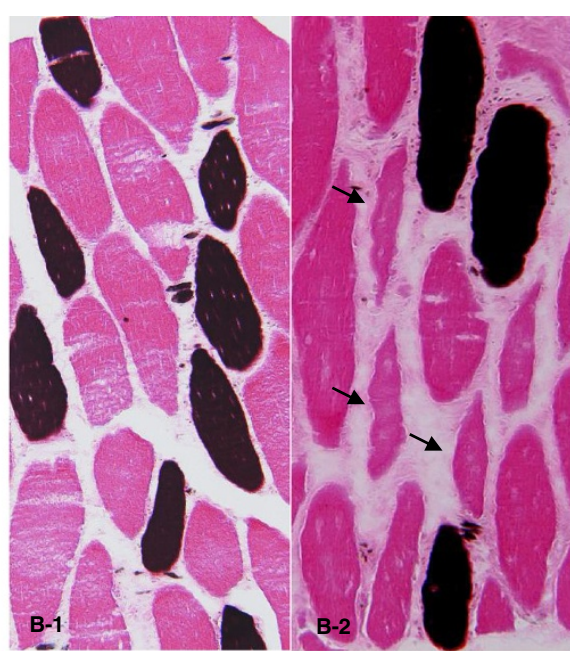

Naive
CIA

Figure 4 Histopathologic feature of quadriceps femoris in NADH-TR and ATPase staining. NADH-TR and ATPase staining in a naive animal (A-1 and B-1) and a CIA animal (A-2 and B-2). In NADH-TR stain, a clear difference in stainability between muscle fibers reflecting the classification into type I and type II muscle fibers was observed in the naive animal; however, a difference in stain ability was not shown and irregularity in the size of muscle fibers was observed in the CIA animal. ATPase staining in CIA animal showed that the atrophic changes in the type II muscle fibers were more significant than those in the type I muscle fibers.

score $(R=0.422$, Figure $2 B)$, arthritis score showed a weak correlation with body weight change [body weight change was calculated with the following formula: body weight change $(\%)$ = body weight at each week $(\mathrm{kg}) /$ body weight before immunization $(\mathrm{kg}) \times 100](\mathrm{R}=-0.610$, Figure $2 \mathrm{C}$ ) and a stronger correlation with creatinine levels $(R=-0.799$, Figure 2D).

\section{Discussion}

In patients with RA, not only pain and dysfunction at joints but also cachexia are observed frequently [31-33], and rheumatoid cachexia is still remains one of major issues. Multiple factors such as disuse atrophy due to joint pain and proinflammatory cytokines such as TNF- $\alpha, \mathrm{IL}-1 \beta$, and IL- 6 are considered attributable to rheumatoid cachexia; however, there have been few reports regarding histopathology of muscle in RA patients and the cause of rheumatoid cachexia is not completely understood yet. In the present study, we sequentially assessed histopathological muscle lesions in a CIA model using CMs. In addition, we investigated the relationships between joint swelling, a major symptom of arthritis,

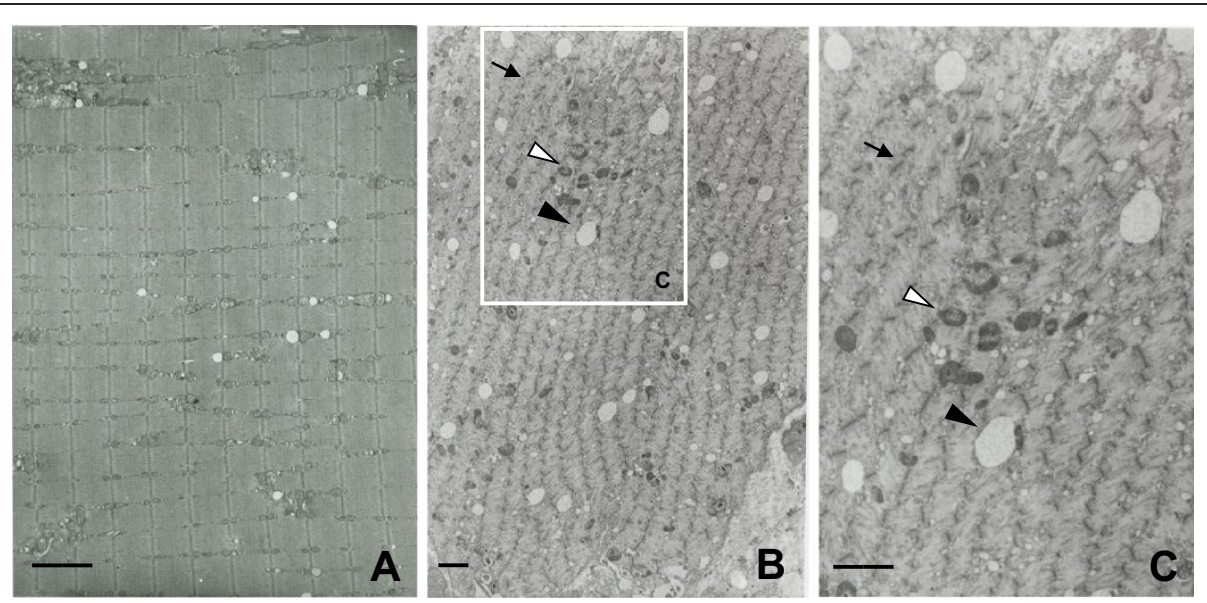

Figure 5 Electron microscopy in quadriceps femoris in naive and $\mathrm{CI} A$ animals. Irregularity of Z-bands $(\uparrow)$ and increases in the number of mitochondria $(\Delta)$ and small vesicles $(\mathbf{A})$ were observed in the CIA animal $(\mathbf{B})$ in comparison with a naive animal $(\mathbf{A})$. $\mathbf{C}$ is high magnification of the region enclosed in the square in $\mathbf{B}$. (bar $2 \mu \mathrm{m})$. 

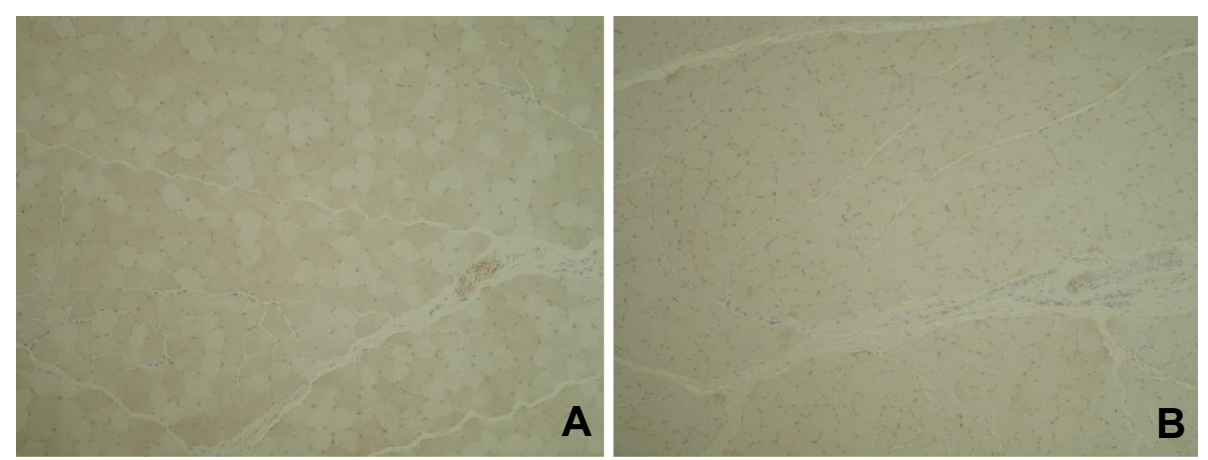

Figure 6 Immunostaining for pimonidazole dosing experiment in quadriceps femoris in naive and $\mathrm{Cl} A$ animals. Mosaic pattern attributable to distribution of type I and II muscle fibers was evident in a naive animal (A); conversely, no similar mosaic pattern was observed in a CIA animal (B).

body weight, and blood chemistry parameters relating to muscle.

In histopathology of HE stained specimens of the quadriceps femoris, necrosis of muscle fibers was observed only in 2 and 1 of 5 animals at Weeks 2 and 3, respectively, and degeneration and atrophy were observed in almost all animals from Week 5. Additionally, similar findings were observed in biceps brachii muscle, longissimus muscle, and gastrocnemius (data not shown), indicating that atrophic change in muscle was induced systemically as shown by the decrease in body weight. Food consumption almost recovered to the range of the values at pre-immunization by Week 9 , but body weight did not completely recover by that time, showing that the decrease in body weight including these histopathological lesions were not considered to be attributable only to malnutrition. In NADH-TR staining, a clear difference in stain ability between muscle fibers reflecting the classification into type I and type II muscle fibers was observed in a naive animal; however, the difference in stain ability was not shown in the CIA animals. Additionally, ATPase staining showed a significant atrophic lesion in the type II muscle fibers but not in the type I muscle fibers. These changes would be related to the disappearance of normal mosaic pattern (that would reflect the regular distribution of the type I and type II muscle fibers) in CIA model which was confirmed in the pimonidazole dosing experiment, and indicated that the normal distribution of partial pressure oxygen had been lost in CIA model. In addition, electron microscopic observation revealed an increase in the number of mitochondria, and this was considered to be a compensatory reaction against hypoxia. The histopathological lesions in the muscle observed in the present study were similar to those of the patients with RA [34]. The study of hypoxia in RA patients showed that hypoxia and HMGB-1 were associated with development of arthritis [27]. On the other hand, physical activity and aerobic exercise ameliorate not only the motor function but also swelling and stiffness of the joints in clinical practice. These reports showed that hypoxia was associated with swelling and malfunction of joints, at least in part. Moreover, the results in the present study indicated that muscle hypoxia was possibly related to the muscle wasting.

It is known that muscle atrophy can be mediated by multiple factors, including glucocorticoids [28-35], and proinflammatory cytokines such as IL- $1 \beta$, TNF- $\alpha$, and IL-6 [35]. Previous studies showed that the expression and activity of the transcription factor $\mathrm{C} / \mathrm{EBP} \beta$ and $\delta$ were increased in the skeletal muscle during sepsis in rats [36]. In the same experiments, treatment with the glucocorticoid receptor antagonist prevented the sepsis-induced activation of $\mathrm{C} / \mathrm{EBP} \beta$ and $\delta$, suggesting that the transcription factors are, at least in part, regulated by glucocorticoids. The expression of these transcription factors, especially that of $\mathrm{C} / \mathrm{EBP} \beta$, was confirmed by western-blot analysis in skeletal muscle obtained from the present study, and it was most significant at Week 5 (data not shown), and a marked increase in serum cortisol concentration was noted at the same time point. These results indicated that cortisol is involved in an increase in expression of $\mathrm{C} / \mathrm{EBP} \beta$ and might be related to the muscle wasting in this CIA model.

Although serum TNF- $\alpha$ was not detected at any time point, significant increases in glucocorticoid and proinflammatory cytokines such as IL-6 were noted in this model, and these findings were consistent with results from clinical practice [37]. Further research is needed to elucidate the direct relationship between hypoxia, glucocorticoid, and proinflammatory cytokines in skeletal muscle since it was not made clear in the present study.

The prevalence of cardiovascular disease (CVD) in patients with RA is higher [38], and the risk of mortality due to CVD is $50 \%$ higher [39] than in the general population. As chronic systemic inflammation has been reported to be related to CVD $[40,41]$, the heart was also examined histopathologically in the present study, but no abnormality 
was observed in this organ in any animal in the present study (data not shown). These results showed that the degree of inflammation noted in this CIA model was not associated with CVD. Generally, the creatinine level in preserved urine is measured for an indirect assessment of muscle quantity in clinical practice [31]. In CMs, the volume of urine depends only on spontaneous urination and is unstable; therefore, serum creatinine was measured instead of urine volume in the present study. The results showed that a positive correlation $(R=0.599)$ was noted between body weight and serum creatinine level. The percentage of body fat was about $5 \%$ in CMs of medium-size, as quantified with the DXA method (data not shown), which is not higher than that in humans. Serum creatinine could be as accurate an indicator of muscle quantity in CMs as it is in humans.

Arthritis score did not show a clear correlation with serum CPK $(R=0.422)$; however, the score showed a negative correlation with the change in body weight based on the value at pre-immunization $(\mathrm{R}=-0.610)$ and a stronger negative correlation with serum creatinine, a marker which reflects muscle mass $(R=-0.799)$. Thus, arthritis score was increased accompanied by a decrease in serum creatinine, a marker to reflect muscle mass, and the results were considered plausible and capable of extrapolation to humans since aging is thought to be one of the risk factors for RA and muscle mass generally decreases with aging. Also in the study using mice CIA model, a similar correlation was noted between muscle weight and arthritis score [42].

\section{Conclusions}

From these results, we concluded that muscle wasting exacerbates joint swelling, and that therapeutic target would not only be the arthritic joints but also the skeletal muscle. The CIA model using CMs has similar characteristics to patients with RA in respect of the changes at the joints and in muscle, suggesting that the CIA monkey model in the present study can be useful for the development of new drugs for human arthritis therapy.

\section{Abbreviations \\ CIA: Collagen-induced arthritis; CM: Cynomolgus monkey; RA: Rheumatoid arthritis; TNF-a: Tumor necrosis factor-a; DMARDs: Disease-modifying antirheumatic drugs; AIA: Adjuvant-induced arthritis; Cll: Bovine type II collagen; FCA: Freund's complete adjuvant; CPK: Creatine phosphokinase; CRP: C-reactive protein; IL-6: Interleukin-6; HE: Hematoxylin-eosin; CVD: Cardiovascular disease.}

\section{Competing interests}

The authors declare that they have no competing interests.

\section{Authors' contributions}

Abeyama contributed to the study design. Horai, Nagaoka, Higuchi, Kasai, Yoshioka, and Umekita contributed to the acquisition of data. Horai, Nagaoka, Higuchi, Fukuzaki, Nagata, Miyata, and Abeyama contributed to the analysis and the interpretation of data. Horai, Miyata, and Abeyama contributed to the manuscript preparation. All authors read and approved the final manuscript.

\section{Acknowledgements}

We thank Dr. Fumio Chatani and Henry Smith for reviewing the manuscript. In addition, we would like to thank the staffs of SNBL DSR for carrying out the in-life part of the study. We are also grateful to Miyuki Matsuda and

Tomohiro Izumi for their valuable technical assistance.

\section{Author details}

'Department of Pharmacology, Kagoshima University Graduate School of Medical and Dental Sciences, 8-35-1 Sakuragaoka, Kagoshima 890-8544, Japan. ${ }^{2}$ Shin Nippon Biomedical Laboratories, Ltd. Drug Safety Research Laboratories (SNBL DSR), 2438 Miyanoura, Kagoshima 891-1394, Japan. ${ }^{3}$ Department of Neurology and Geriatrics, Kagoshima University Graduate School of Medical and Dental Sciences, 8-35-1 Sakuragaoka, Kagoshima 890-8544, Japan. ${ }^{4}$ Department of Tumor Pathology, Kagoshima University Graduate School of Medical and Dental Sciences, 8-35-1 Sakuragaoka, Kagoshima 890-8544, Japan. ${ }^{5}$ Division of Organ Pathology, Faculty of Medicine, Tottori University, 86 Nishi-machi, Yonago, Tottori 683-8503, Japan. ${ }^{6}$ Department of Radiology, Morizono Hospital, 19-38 Oshoji-cho, Satsumasendai, Kagoshima 895-0076, Japan.

Received: 16 January 2013 Accepted: 5 July 2013

Published: 9 July 2013

\section{References}

1. Uchiyama Y, Yorozu K, Hashizume M, Moriya Y, Mihara M: Tocilizumab, a humanized anti-interleukin-6 receptor antibody, ameliorates joint swelling in established monkey collagen-induced arthritis. Biol Pharm Bull 2008, 31:1159-1163 [Pubmed].

2. Kato A, Matsuo S, Takai H, Uchiyama Y, Mihara M, Suzuki: Early effects of tocilizumab on bone and bone marrow lesions in a collagen-induced arthritis monkey model. Exp Mol Pathol 2008, 84:262-270 [Pubmed].

3. Saito M, Yoshigae Y, Nakayama J, Ogawa Y, Ohtsuki M, Kurihara A, Ikeda T: Tissue distribution of humanized anti-human Fas monoclonal antibody (R-125224) based on fas antigen-antibody reaction in collagen-induced arthritis monkeys. Life Sci 2007, 80:2005-2014 [Pubmed].

4. Sacre SM, Andreakos E, Taylor P, Feldmann M, Foxwell BM: Molecular therapeutic targets in rheumatoid arthritis. Expert Rev Mol Med 2005, 7:1-20 [Pubmed].

5. Yang C, Robbins PD: Immunosuppressive exosomes: a new approach for treating arthritis. Int I Rheumatol 2012, 2012:8. 573528. [Pubmed].

6. Ogasawara A, Utoh M, Nii K, Ueda A, Yoshikawa T, Kume T, Fukuzaki K: Effect of oral ketoconazole on oral and intravenous pharmacokinetics of simvastatin and its acid in cynomolgus monkeys. Drug Metab Dispos 2009, 37:122-128 [Pubmed].

7. Carr B, Norcross R, Fang Y, Lu P, Rodrigues AD, Shou M, Rushmore T, Booth-Genthe C: Characterization of the rhesus monkey CYP3A64 enzyme: species comparisons of CYP3A substrate specificity and kinetics using baculovirus-expressed recombinant enzymes. Drug Metab Dispos 2006, 34:1703-1712 [Pubmed].

8. Prueksaritanont T, Kuo Y, Tang C, Li C, Qiu Y, Lu B, Strong-Basalyga K, Richards $\mathrm{K}, \mathrm{Carr} \mathrm{B}, \mathrm{Lin} \mathrm{JH}$ : In vitro and in vivo CYP3A64 induction and inhibition studies in rhesus monkeys: a preclinical approach for CYP3A-mediated drug interaction studies. Drug Metab Dispos 2006, 34:1546-1555 [Pubmed].

9. Lin HS, Hu CY, Chan HY, Liew YY, Huang HP, Lepescheux L, Bastianelli E, Baron R, Rawadi G, Clément-Lacroix P: Anti-rheumatic activities of histone deacetylase (HDAC) inhibitors in vivo in collagen-induced arthritis in rodents. Br J Pharmacol 2007, 150:862-872 [Pubmed].

10. Fujii Y, Hirayama T, Ohtake H, Ono N, Inoue T, Sakurai T, Takayama T, Matsumoto K, Tsukahara N, Hidano S, Harima N, Nakazawa K, Igarashi Y, Goitsuka R: Amelioration of collagen-induced arthritis by a novel S1P1 antagonist with immunomodulatory activities. J Immunol 2012, 188:206-215 [Pubmed].

11. Sato T, Fujii R, Konomi K, Yagishita N, Aratani S, Araya N, Aono H, Yudoh K, Suzuki N, Beppu M, Yamano Y, Nishioka K, Nakajima T: Overexpression of SPACIA1/SAAL1, a newly identified gene that is involved in synoviocyte proliferation, accelerates the progression of synovitis in mice and humans. Arthritis Rheum 2011, 63:3833-3842 [Pubmed].

12. Zhang W, Wang B, Wang F, Zhang J, Yu J: CTLA4-FasL fusion product suppresses proliferation of fibroblast-like synoviocytes and progression of adjuvant-induced arthritis in rats. Mol Immunol 2012, 50:150-159 [Pubmed].

13. Chou LW, Wang J, Chang PL, Hsieh YL: Hyaluronan modulates accumulation of hypoxia-inducible factor-1 alpha, inducible nitric oxide 
synthase, and matrix metalloproteinase- 3 in the synovium of rat adjuvant-induced arthritis model. Arthritis Res Ther 2011, 13:R90 [Pubmed].

14. Schierbeck H, Lundbäck P, Palmblad K, Klevenvall L, Erlandsson-Harris H, Andersson U, Ottosson L: Monoclonal anti-HMGB1 (high mobility group box chromosomal protein 1) antibody protection in two experimental arthritis models. Mol Med 2011, 17:1039-1044 [Pubmed]

15. Abreu JR, Krausz S, Dontje W, Grabiec AM, de Launay D, Nolte MA, Tak PP, Reedquist KA: Sustained T cell Rap1 signaling is protective in the collagen-induced arthritis model of rheumatoid arthritis. Arthritis Rheum 2010, 62:3289-3299 [Pubmed].

16. Wierda D, Smith HW, Zwickl CM: Immunogenicity of biopharmaceuticals in laboratory animals. Toxicology 2001, 158:71-74 [Pubmed].

17. Vierboom MP, Breedveld E, Kondova I, t Hart BA: Collagen-induced arthritis in common marmosets: a new nonhuman primate model for chronic arthritis. Arthritis Res Ther 2010, 12:R200 [Pubmed].

18. Chapman K, Pullen N, Graham M, Ragan I: Preclinical safety testing of monoclonal antibodies: the significance of species relevance. Nat Rev Drug Discov 2007, 6:120-126 [Pubmed].

19. Crowson CS, Matteson EL, Roger VL, Therneau TM, Gabriel SE: Usefulness of risk scores to estimate the risk of cardiovascular disease in patients with rheumatoid arthritis. Am J Cardiol 2012, 110:420-424 [Pubmed].

20. Bisoendial RJ, Stroes ES, Tak PP: Critical determinants of cardiovascular risk in rheumatoid arthritis. Curr Pharm Des 2011, 17:21-26 [Pubmed].

21. Roubenoff R, Roubenoff RA, Cannon JG, Kehayias JJ, Zhuang H, Dawson-Hughes B, Dinarello CA, Rosenberg $H$ : Rheumatoid cachexia: cytokine-driven hypermetabolism accompanying reduced body cell mass in chronic inflammation. J Clin Invest 1994, 93:2379-2386 [Pubmed].

22. Summers GD, Metsios GS, Stavropoulos-Kalinoglou A, Kitas GD: Rheumatoid cachexia and cardiovascular disease. Nat Rev Rheumatol 2010, 6:445-451 [Pubmed].

23. Walsmith J, Roubenoff R: Cachexia in rheumatoid arthritis. Int J Cardiol 2002, 85:89-99 [Pubmed].

24. Plasqui G: The role of physical activity in rheumatoid arthritis. Physiol Behav 2008, 94:270-275 [Pubmed].

25. Hollander AP, Corke KP, Freemont AJ, Lewis CE: Expression of hypoxia-inducible factor 1alpha by macrophages in the rheumatoid synovium: implications for targeting of therapeutic genes to the inflamed joint. Arthritis Rheum 2001, 44:1540-1544 [Pubmed].

26. Peters CL, Morris CJ, Mapp PI, Blake DR, Lewis CE, Winrow VR: The transcription factors hypoxia-inducible factor 1alpha and Ets-1 co localize in the hypoxic synovium of inflamed joints in adjuvant-induced arthritis. Arthritis Rheum 2004, 50:291-296 [Pubmed]

27. Hamada T, Torikai M, Kuwazuru A, Tanaka M, Horai N, Fukuda T, Yamada S, Nagayama S, Hashiguchi K, Sunahara N, Fukuzaki K, Nagata R, Komiya S, Maruyama I, Fukuda T, Abeyama K: Extracellular high mobility group box chromosomal protein 1 is a coupling factor for hypoxia and inflammation in arthritis. Arthritis Rheum 2008, 58:2675-2685 [Pubmed]

28. Hasselgren PO: Glucocorticoids and muscle catabolism. Curr Opin Clin Nutr Metab Care 1999, 2:201-205 [Pubmed].

29. Menconi M, Fareed M, O'Neal P, Poylin V, Wei W, Hasselgren PO: Role of glucocorticoids in the molecular regulation of muscle wasting. Crit Care Med 2007, 35:S602-S608 [Pubmed].

30. Schakman O, Gilson H, Thissen JP: Mechanisms of glucocorticoid-induced myopathy. J Endocrinol 2008, 197:1-10 [Pubmed].

31. Summers GD, Deighton CM, Rennie MJ, Booth AH: Rheumatoid cachexia: a clinical perspective. Rheumatology (Oxford) 2008, 47:1124-1131 [Pubmed].

32. Rall LC, Walsmith JM, Snydman L, Reichlin S, Veldhuis JD, Kehayias JJ, Abad LW, Lundgren NT, Roubenoff R: Cachexia in rheumatoid arthritis is not explained by decreased growth hormone secretion. Arthritis Rheum 2002, 46:2574-2577 [Pubmed].

33. Rall LC, Roubenoff R: Rheumatoid cachexia: metabolic abnormalities, mechanisms and interventions. Rheumatology (Oxford) 2004, 43:1219-1223 [Pubmed].

34. Magyar E, Talerman A, Mohacsy J, Wouters HW, de Bruijn WC: Muscle change in rheumatoid arthritis. A review of the literature with a study of 100 cases. Virchows Arch A Path Anat Histol 1977, 373:267-278 [Pubmed].

35. Saini A, Al-Shanti N, Stewart CE: Waste management - cytokines, growth factors and cachexia. Cytokine Growth Factor Rev 2006, 17:475-486 [Pubmed].

36. Penner G, Gang G, Sun X, Wray C, Hasselgren PO: C/EBP DNA-binding activity is upregulated by a glucocorticoid-dependent mechanism in septic muscle. Am J Physiol Regul Integr Comp Physiol 2002, 282:R439-R444 [Pubmed].
37. Nishimoto N, Terao K, Mima T, Nakahara H, Takagi N, Kakehi T: Mechanisms and pathologic significances in increase in serum interleukin-6 (IL-6) and soluble IL-6 receptor after administration of an anti-IL-6 receptor antibody, tocilizumab, in patients with rheumatoid arthritis and Castleman disease. Blood 2008, 112:3959-3964 [Pubmed].

38. Solomon DH, Karlson EW, Rimm EB, Cannuscio CC, Mandl LA, Manson JE, Stampfer MJ, Curhan GC: Cardiovascular morbidity and mortality in women diagnosed with rheumatoid arthritis. Circulation 2003, 107:1303-1307 [Pubmed].

39. Aviña-Zubieta JA, Choi HK, Sadatsafavi M, Etminan M, Esdaile JM, Lacaille D: Risk of cardiovascular mortality in patients with rheumatoid arthritis: a meta-analysis of observational studies. Arthritis Rheum 2008, 59:1690-1697 [Pubmed].

40. Maradit-Kremers H, Nicola PJ, Crowson CS, Ballman KV, Gabriel SE: Cardiovascular death in rheumatoid arthritis: a population-based study. Arthritis Rheum 2005, 52:722-732 [Pubmed].

41. Libby $P$, Ridker PM, Maseri A: Inflammation and atherosclerosis. Circulation 2002, 105:1135-1143 [Pubmed]

42. Hartog A, Hulsman J, Garssen J: Locomotion and muscle mass measures in a murine model of collagen-induced arthritis. BMC Musculoskelet Disord 2009, 10:59 [Pubmed].

doi:10.1186/1471-2474-14-205

Cite this article as: Horai et al:: Muscle wasting associated with pathologic change is a risk factor for the exacerbation of joint swelling in collagen-induced arthritis in cynomolgus monkeys. BMC Musculoskeletal Disorders 2013 14:205.

\section{Submit your next manuscript to BioMed Central and take full advantage of:}

- Convenient online submission

- Thorough peer review

- No space constraints or color figure charges

- Immediate publication on acceptance

- Inclusion in PubMed, CAS, Scopus and Google Scholar

- Research which is freely available for redistribution

Submit your manuscript at www.biomedcentral.com/submit
C BioMed Central 